\title{
RELACIÓN DE LA INDUSTRIA FARMACÉUTICA CON LOS MÉDICOS RESIDENTES DE LA REPÚBLICA DOMINICANA
}

\section{Relationship of the Pharmaceutical Industry with the Medical Residences of the Dominican Republic}

\section{Ricardo Elías Melgen y César Mota Curiel}

Recibido: 16 de enero, 2020 • Aprobado: 12 mayo, 2020

Cómo citar: Elías Melgen R, Mota Curiel C. Relación de la industria farmacéutica con los médicos residentes de la República Dominicana. cysa [Internet]. 18 de septiembre de 2020 [citado 18 de septiembre de 2020];4(3):33-41. Disponible en: https://revistas. intec.edu.do/index.php/cisa/article/view/1921

\section{Resumen}

Introducción: las relaciones médicas con la industria farmacéutica son cada vez más complejas y los desafíos éticos que plantea son reales.

Objetivo: conocer la relación que existe entre la industria farmacéutica con los médicos residentes del país y el impacto que pudiera tener esta relación.

Material y Métodos: estudio descriptivo transversal mediante encuesta electrónica anónima. Se consideró la participación si confirmaban el llenado de la encuesta, que estuvo disponible durante abril y mayo de 2019. Se realizó un análisis descriptivo y se aseguró la confidencialidad y el anonimato de los datos.

Resultados: se encuestaron 77 médicos residentes del país; $55.8 \%$ recibe semanalmente visita del representante farmacéutico. Al menos cinco veces al año recibieron obsequio $(49.4 \%)$, participaron en comida, cena u otro evento

\footnotetext{
a Departamento de Neumología, Hospital Infantil Dr. Robert Reid Cabral, Centro Nacional de Investigación en Salud Materno Infantil Dr. Hugo Mendoza (CENISMI), República Dominicana. Correo-e: emelgen@hirrc.gov.do, reliasmelgen@yahoo.es ORCID: 0000-0001-7901-0532
}

\begin{abstract}
Introduction: Medical relationships with the pharmaceutical industry are increasingly complex and the ethical challenges it poses are real.

Objective: To know the relationship that exists between the pharmaceutical industry and the resident doctors of the country and the impact that this relationship could have.

Material and Methods: Cross-sectional descriptive study, through anonymous electronic survey participation was considered if they confirmed the completion of the survey that was available during April and May 2019. A descriptive analysis was conducted and the confidentiality and anonymity of the data was ensured.

Results: 77 resident physicians of the country were surveyed; $55.8 \%$ receive a weekly visit from the pharmaceutical representative. At least 5 times a year they received a gift $(49.4 \%)$, participated in a meal, dinner or other social event $(48.1 \%)$, participated in a talk funded

\footnotetext{
b Departamento de Emergencias y Urgencias, Hospital Infantil Dr. Robert Reid Cabral, República Dominicana.
}

Correo-e: cmota_curiel@hotmail.com
\end{abstract}


social (48.1\%), participó en charla financiada por empresa farmacéutica $(57.1 \%) .40 .3 \%$ recibieron una vez al año el pago por parte de la industria farmacéutica, de inscripción/ viaje a algún evento (congreso, jornada, etc.).

Están de acuerdo en que los regalos/propuestas de la industria farmacéutica influyen en la prescripción de los medicamentos (20.8\%), considera bien aceptar pago de eventos ( $45.4 \%)$, refirieron que en su residencia se suelen aceptar regalos y ofertas y no ha tenido un espacio formativo sobre relaciones con la industria farmacéutica $(89.6 \%)$.

Conclusión: existe un alto nivel de contacto de los médicos residentes con la industria farmacéutica, es necesario más y mejor formación en manejo de conflictos de intereses en la práctica profesional.

Palabras clave: industria farmacéutica; prescripción de medicamentos; responsabilidad social; publicidad de medicamento; especialidad médica; ética médica.

\section{Introducción}

La importancia de la industria farmacéutica (IF) es incuestionable en el desarrollo de nuevos tratamientos, productos y medicamentos para mejorar la calidad de vida y restablecer la salud de las personas; pero existe una estrecha y delicada relación con los profesionales de la salud, que puede llevar de uno y otro extremo a conflictos de interés, en el que el perjudicado final puede ser el paciente. La selección de un medicamento por parte del médico es un acto complejo, que puede estar influido directa o indirectamente por la opinión de colegas, publicaciones científicas y sobre todo por estrategias de mercadeo y las actividades de promoción que realiza la IF; esta práctica de promoción influye en los patrones de prescripción del médico y en el consumo de medicamentos, pudiendo generar conflictos de intereses que alteren la decisión clínica. ${ }^{1}$

La frecuente interacción entre la IF con los médicos ha sido documentada, así como los tipos de beneficios que estos generalmente reciben, y si bien se reconoce que estas actividades de promoción by a pharmaceutical company (57.1\%). 40.3\% received once a year the payment by the FI, of registration / trip to an event (congress, day, etc.).

They agree that the gifts / proposals of the pharmaceutical industry influence the prescription of the medicines (20.8\%), consider accepting payment of events (45.4\%), they said that in their residence they usually accept gifts and offers of the pharmaceutical industry and has not had a training space on relations with the pharmaceutical industry (89.6\%).

Conclusion: There is a high level of contact of resident physicians with the Pharmaceutical Industry, it is necessary more and better training in handling conflicts of interest in professional practice.

Keywords: Pharmaceutical industry; drug prescriptions; social responsibility; drug advertising; medical speciality; medical ethics.

influyen en la prescripción y elevan el costo final de los productos, encuentran como apropiado el recibir esos beneficios. ${ }^{2,3}$

Existen legislaciones y reglamentaciones en donde se regulan los aspectos relacionados con la transparencia de la promoción farmacéutica, estableciendo que la IF no debe condicionar ni incentivar a los médicos mediante prácticas poco éticas, ya sea ofreciendo cursos, viajes, premios, regalos u otros. ${ }^{4-6}$ Médicos y representantes de empresas farmacéutica han sido sancionados judicialmente por haber compensado ilícitamente a los médicos que accedieron a recomendar la prescripción de los productos de la compañía, en desmedro de otros. ${ }^{7}$

En el 1998 la Comisión Central de Deontología de la Organización Médica Colegial (OMC) de España, con relación a la libertad de prescripción, afirmó que el principal compromiso ético del médico consiste en prestar a sus pacientes, y con el consentimiento de estos, el mejor servicio que sea capaz, tal como dictan la ética profesional y el buen juicio clínico; esto incluye y exige actuar 
con la responsabilidad que viene de la mano de la libertad de prescripción. ${ }^{8}$

El objetivo de la presente investigación es conocer la relación que existe entre la industria farmacéutica con los médicos residentes del país y el impacto que pudiera tener esta relación.

\section{Material y método}

Estudio descriptivo transversal, mediante encuesta electrónica a los Médicos Residentes de las diferentes residencias de República Dominicana, para conocer la relación que existe entre la IF con los médicos residentes del país y el impacto que pudiera tener esta relación.

Se elaboró un cuestionario estructurado en cinco bloques: 1) información a los participantes sobre el objeto del estudio y la solicitud a participar, 2) Frecuencia y características de las interacciones de los residentes con la IF, 3) Percepción y actitud de los residentes en relación a la interacción con la IF, 4) Percepción del entorno de los residentes en relación a la interacción con la IF y 5) Conocimiento del marco regulatorio.

Se utilizó google.form como herramienta para elaborar y enviar la encuesta; previo a su envío masivo, se realizó un pilotaje con cinco residentes; luego de la validación, el cuestionario fue enviado vía WhatsApp a través de los grupos de residencias médicas del país y/o de forma personal; se consideró la aceptación y participación si confirmaban el llenado de la encuesta. La encuesta estuvo disponible durante abril y mayo de 2019. Se realizó un análisis descriptivo y se aseguró la confidencialidad y el anonimato de los datos.

\section{Resultados}

Un total de 77 médicos residentes aceptaron completar la encuesta; en el gráfico 1 se muestra que el $44.2 \%$ de los encuestados estaban realizando la residencia en pediatría, $13 \%$ en Emergencias y Cuidados Intensivos, $14.2 \%$ en Ginecología y Obstetricia, $7.8 \%$ otras especialidades médicas y el $20.8 \%$ no respondió cuál residencia estaba realizando. Asimismo, el $20.7 \%$ cursaba el primer año de residencias, $14.3 \%$ el segundo año, $26 \%$ el tercer año, $27.3 \%$ el cuarto año y el $11.7 \%$ cursaba un quinto año o más.

Gráfico 1. Distribución de los encuestados por especialidad y año de residencia
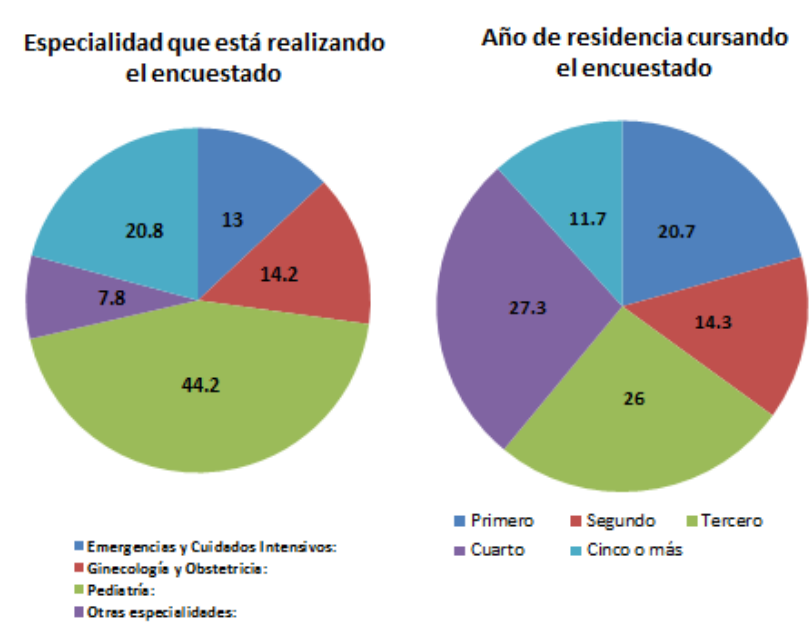

Fuente: elaboración propia.

$\mathrm{Al}$ medir la frecuencia y características de las interacciones de los residentes encuestados con la IF (tabla 1), se puede observar que el $55.8 \%$ de los encuestados recibe con una frecuencia semanal la visita de un representante farmacéutico, y el $23.4 \%$ de forma mensual; asimismo, el $51.9 \%$ refiere que semanalmente recibe de forma gratuita un fármaco, y el $23.4 \%$ lo recibe mensual. Solo el $2.6 \%$ y 3.9 $\%$ de los encuestados refirió que nunca ha recibido la visita de un representante farmacéutico o muestras gratuitas de un fármaco, respectivamente.

El $49.4 \%$ de los residentes encuestados, refirieron que al menos cinco veces al año reciben algún obsequio por parte de la IF como un pequeño regalo no relacionado con la formación (bolígrafo, libreta, etc.) y el $10.3 \%$ este obsequio lo recibe mensual. El $70.1 \%$ refirió nunca haber recibido como obse- 
quio un libro o material de trabajo (estetoscopio, etc.) y el $24.7 \%$ refirió haberlos recibido una vez al año.

Se evidencia que el $48.1 \%$ de los encuestados refirieron que al menos cinco veces en el año, habían participado en una comida, cena $\mathrm{u}$ otro evento social patrocinado por la IF y solo el $11.6 \%$ refirió nunca haber participado en este tipo de eventos; igualmente el 57.1 \% refirió haber participado, al menos cinco veces en el año, en alguna charla financiada por una empresa farmacéutica, y solo el $13 \%$ refirió que nunca ha participado en estos eventos.

Con relación al pago por parte de la IF, de inscripción/viaje a algún evento (congreso, jornada, etc.), el $40.3 \%$ de los residentes encuestados lo habían recibido una vez al año y el $54.5 \%$ manifestó que nunca lo había recibido; así mismo el 96.1% refirió que nunca ha obtenido beca o premio y el $91 \%$ de los encuestados nunca ha participado en una investigación financiada por la IF.

\section{Tabla 1. Frecuencia y características de las interacciones de los residentes con la industria farmacéutica}

\begin{tabular}{|c|c|c|c|c|c|}
\hline & $\begin{array}{l}\text { Semanal } \\
\%\end{array}$ & $\begin{array}{l}\text { Mensual } \\
\%\end{array}$ & $\begin{array}{c}\text { al menos } 5 \\
\text { veces al año } \\
\%\end{array}$ & $\begin{array}{c}\text { una vez al } \\
\text { ańo } \\
\%\end{array}$ & $\begin{array}{l}\text { Nunca } \\
\%\end{array}$ \\
\hline $\begin{array}{l}\text { Qué tan frecuente recibe la visita de un } \\
\text { representante farmacéutico }\end{array}$ & 55.8 & 23.4 & 18.2 & 0.0 & 2.6 \\
\hline Muestra gratuita de un fármaco & 51.9 & 23.4 & 20.8 & 0.0 & 3.9 \\
\hline $\begin{array}{l}\text { Ha recibido un pequeño regalo no relacionado con forma- } \\
\text { ción (bolígrafo, libreta, etc.) }\end{array}$ & 0.0 & 10.3 & 49.4 & 22.1 & 18.2 \\
\hline Libro o material de trabajo (estetoscopio, etc.) & 0.0 & 0.0 & 5.2 & 24.7 & 70.1 \\
\hline Comida, cena u otro evento social & 0.0 & 19.5 & 48.1 & 20.8 & 11.6 \\
\hline Charla financiada por una empresa farmacéutica & 0.0 & 6.5 & 57.1 & 23.4 & 13 \\
\hline $\begin{array}{l}\text { Pago de inscripción/viaje a algún evento } \\
\text { (congreso, jornada, etc.) }\end{array}$ & 0.0 & 0.0 & 5.2 & 40.3 & 54.5 \\
\hline
\end{tabular}

\section{Fuente: elaboración propia.}

$\mathrm{Al}$ medir la percepción y actitud de los residentes con relación a la interacción con la IF, en la tabla 2 , se observa que un $20.8 \%$ de los encuestados están de acuerdo en que los regalos/propuestas de la IF influyen en la prescripción de los medicamentos y el $45.5 \%$ está en desacuerdo. Asimismo, el $22.1 \%$ está de acuerdo en que la financiación de la IF a las sociedades científicas influye en sus actividades, y el $32.5 \%$ está en desacuerdo.

Se observa además que el $45.4 \%$ considera bien aceptar pago de eventos por la IF y solo $18.2 \%$ está en desacuerdo con estos pagos; el $40.3 \%$ entiende que el hospital debería controlar las visitas de representantes farmacéuticos. 
Tabla 2. Percepción y actitud de los residentes con relación a la interacción con la industria farmacéutica

\begin{tabular}{|c|c|c|c|c|}
\hline & $\begin{array}{c}\text { De } \\
\text { acuerdo } \\
\%\end{array}$ & $\begin{array}{c}\text { Desacuerdo } \\
\%\end{array}$ & $\begin{array}{c}\text { Neutral } \\
\%\end{array}$ & $\begin{array}{c}\text { No } \\
\text { responde } \\
\%\end{array}$ \\
\hline $\begin{array}{c}\text { Los regalos/propuestas de la IF influyen en mi } \\
\text { prescripción }\end{array}$ & 20.8 & 45.5 & 33.7 & 0.0 \\
\hline $\begin{array}{c}\text { La financiación recibida de la IF por las } \\
\text { sociedades científicas influye en sus actividades }\end{array}$ & 22.1 & 32.5 & 45.4 & 0.0 \\
\hline $\begin{array}{c}\text { Considera bien aceptar pago de eventos por la IF } \\
\text { El hospital debería controlar las visitas de } \\
\text { representantes farmacéuticos }\end{array}$ & 45.4 & 18.2 & 35.1 & 1.3 \\
\hline \multicolumn{2}{c|}{40.3} & 27.3 & 28.6 & 3.8 \\
\hline
\end{tabular}

Fuente: elaboración propia

El $63.6 \%$ de los encuestados refirieron que en su residencia se suelen aceptar regalos y ofertas de la IF, a pesar de que el $42.8 \%$ de las residencias no facilita el acceso a regalos y ofertas de la IF; un 45.4 $\%$ refirieron que hay médicos preocupados por la relación con la IF. (Tabla 3)

Tabla 3. Percepción del entorno de los residentes en relación a la interacción con la industria farmacéutica

\begin{tabular}{|l|c|c|c|}
\hline & $\begin{array}{c}\text { No } \\
\%\end{array}$ & $\begin{array}{c}\text { Sí } \\
\%\end{array}$ & $\begin{array}{c}\text { No me he } \\
\text { percatado } \\
\%\end{array}$ \\
\hline En mi residencia se suelen aceptar regalos y ofertas de la IF & 5.2 & 63.6 & 31.2 \\
\hline Mi residencia facilita el acceso a regalos y ofertas de la IF & 42.8 & 24.7 & 32.5 \\
\hline $\begin{array}{l}\text { En mi residencia encuentro médicos preocupados por la } \\
\text { relación con la IF }\end{array}$ & 31.2 & 45.4 & 23.4 \\
\hline
\end{tabular}

Fuente: elaboración propia

El $27.3 \%$ de los encuestados no conoce y el $61 \%$ no sabe si existe normativa en su trabajo que regule la interacción con la IF; el $89.6 \%$ no ha tenido un espacio formativo sobre relaciones con la IF y el $79.2 \%$ considera necesaria más formación sobre relaciones con la IF (tabla 4). El $34 \%$ de los residentes encuestados consideran como insuficiente la formación recibida en búsqueda de evidencia terapéutica. 
Tabla 4. Conocimiento del marco regulatorio

\begin{tabular}{|l|c|c|c|}
\hline & No & Si & No se \\
\% & \% \\
\hline $\begin{array}{l}\text { Conoce si existe normativa en su } \\
\text { trabajo que regule la interacción } \\
\text { con la IF }\end{array}$ & 27.3 & 11.7 & 61 \\
\hline $\begin{array}{l}\text { Ha tenido un espacio formativo } \\
\text { sobre relaciones con la IF }\end{array}$ & 89.6 & 10.4 & 0.0 \\
\hline $\begin{array}{l}\text { Considera necesario más forma- } \\
\text { ción sobre relaciones con la IF }\end{array}$ & 9.1 & 79.2 & 11.7 \\
\hline
\end{tabular}

Fuente: elaboración propia

\section{Discusión}

Es un deber y obligación de todo médico prescribir con responsabilidad y moderación a la hora de prevenir, diagnosticar y tratar la enfermedad. La importancia de la IF para mejorar la calidad de vida y restablecer la salud de las personas es incuestionable, independientemente del hecho de que como industria se tenga un interés económico, es el médico quien no puede perder de vista que su compromiso es con el bienestar del paciente y no con la IF. ${ }^{9-11}$

Hemos visto como las prácticas de promoción y mercadeo de la IF generan una alta interacción con los médicos, y que de alguna manera podrían influir en los patrones de prescripción y consumo de medicamentos. Es importante tener presente los aspectos éticos relacionados con los conflictos de intereses del médico que acepta o solicita regalos u otro tipo de relaciones económicas con cualquier empresa que provee insumos médicos, especialmente con la IF, donde es más factible que la presencia de conflictos de intereses pueda influir el acto de prescribir.
Esta alta interacción entre la IF y médicos ha sido documentada ampliamente, hasta el $86 \%$ de médicos encuestados refieren recibir muestras médicas con elevada frecuencia, $39 \%$ útiles de escritorio, $19 \%$ inscripciones a congresos y $12 \%$ invitaciones a comer; si bien estos médicos reconocen que las actividades de promoción influyen en la prescripción y elevan el costo final de los productos, encuentran apropiado recibir esos beneficios. ${ }^{2}$ En una encuesta realizada a médicos residentes en España, el $98 \%$ refirió haber tenido relación con la IF, solo el $20 \%$ creía que influía en su prescripción, pero el $48 \%$ refería que esta relación influía en los demás médicos, ${ }^{12}$ algo así como yo no me dejo influenciar pero el otro sí; también se ha evidenciado, que el estilo de relación entre los médicos, la IF y sus representantes influye en la conducta prescriptora, existiendo deferencias entre lo que el profesional considera que debe hacerse y lo que se hace realmente en las relaciones con la IF. ${ }^{13}$

La IF realiza diversos eventos que tiene una aparente finalidad científica; llevan como invitado especial a un médico cuyo rol es dirigir una conferencia, con el objetivo de destacar los beneficios que patrocina el evento. En muchas ocasiones, son los propios médicos quienes, permiten que los laboratorios farmacéuticos establezcan los programas científicos 0 académicos. ${ }^{14}$

Con la presente investigación, hemos podido evidenciar que existe una alta frecuencia de interacción entre la IF y los médicos residentes dominicanos, quienes semanalmente reciben la visita de un representante en el $55.8 \%$ de los encuestados, recibiendo de forma gratuita un fármaco en el $51.9 \%$. El 49.4 $\%$ de los residentes encuestados, refirió que al menos cinco veces por año habían recibido algún obsequio no relacionado con su formación (bolígrafo, libreta, etc.) y más del $70 \%$, nunca ha recibido como obsequio un libro o material de trabajo relacionado con su profesión (estetoscopio, etc.). 
$\mathrm{Al}$ menos 5 veces por año, el $48.1 \%$ de los encuestados refirieron haber participado en una comida, cena $\mathrm{u}$ otro evento social patrocinado por la IF y el $57.1 \%$ asistieron a alguna charla financiada por una empresa farmacéutica. El $40.3 \%$ de los residentes encuestados habían recibido al menos una vez al año el pago por parte de la IF de inscripción/ viaje a algún evento (congreso, jornada, etc.).

Ante la pregunta a médicos del Perú ¿existe conflicto de tipo ético entre la promoción farmacéutica y los médicos al prescribir medicamentos? El 44,8 $\%$ consideró que no existía ningún conflicto ético entre la promoción realizada por la industria farmacéutica y la prescripción de dichos medicamentos ( $47,3 \%$ en mujeres y $43,3 \%$ en varones)..$^{15}$

Es interesante hacer notar la percepción y actitud que los residentes dominicanos tienen con relación a la interacción con la IF, solo el $20.8 \%$ de los encuestados está de acuerdo con que los regalos/ propuestas de la IF influyen en la prescripción de los medicamentos, el $22.1 \%$ entiende que la financiación de la IF a las sociedades científicas influye en las actividades y $45.4 \%$ considera bien aceptar pago de eventos por la IF.

Con base en estos resultados, se hace necesario insistir en la necesidad de actuar reconociendo la existencia de estos conflictos de intereses y de que los médicos residentes son susceptibles de ser influidos por las estrategias de mercadeo de la IF, es por eso que la solicitud de beneficios personales por parte de los médicos residentes debe considerarse con suma prudencia.

El $42.8 \%$ de los encuestados, afirmaron que en sus residencias no se facilita el acceso a regalos y ofertas de la IF, pero un alto porcentaje $(63.6 \%)$ refirieron que suelen aceptarlo. Si bien es cierto, que el 45.4 $\%$ de los residentes encuestados, observó que hay médicos preocupados por la relación con la IF, un $27.3 \%$, no conoce y el $61 \%$ no sabe si existe normativa en su trabajo que regule la interacción con la IF. El 79.2 \% de los residentes encuestados considera necesaria más formación sobre relaciones con la IF, ya que a nivel de las diferentes residencias médicas no se contempla un espacio formativo sobre esta relación médico - IF.

Podemos concluir que la población estudiada utiliza, en una proporción significativa, los beneficios que resultan de la interacción con la IF, a pesar de la evidencia disponible, que señala los inconvenientes y conflictos que esta relación podría traer; es por eso que se hace necesario reglamentar estas interacciones en los médicos en formación (residentes); quienes, por hallarse en una etapa en la cual se fijan patrones de prescripción difíciles de modificar luego, son más susceptibles de ser influidos.

\section{Conflicto de intereses}

Los autores declaran no tener conflictos de intereses, no tener ningún tipo de relaciones con la IF, ni recibir apoyo económico alguno para realizar la presente investigación.

\section{Bibliografía}

1. Castresana L, Mejia R, Aznar M. Actitud de los médicos frente a las prácticas de promoción de la industria farmacéutica. MEDICINA 2005;65:247-251. Disponible en https:// www.medicinabuenosaires.com/demo/revistas/ vol65-05/3/ACTITUD\%20DE\%20LOS\%20 MEDICOS\%20FRENTE\%20A\%20LAS\%20 PRACTICAS\%20DE\%20PROMOCION. pdf. [Revisado 26 febrero 2020].

2. Martín-Aranda P, Vázquez I, Rodríguez de Cossío A. Médicos residentes e industria farmacéutica. Aten Primaria. 2007;39(9). Disponible en https://www.elsevier.es/es-revista-atencion-primaria-27-articulo-medicos-residentes-e-industria-farmaceutica-13109507 [Revisado 26 febrero 2020]. 
3. González-Rubioa R, Escortell-Mayorb E, Del Cura González I. Industria farmacéutica y formación sanitaria especializada: percepciones de los MIR en Madrid. Gac Sanit. 2019;33(2):112-8. Disponible en https://www.sciencedirect.com/ science/article/pii/S0213911117301954 [revisado 26 febrero 2020].

4. Florián CastroR.¿Eséticala promociónfarmacéutica en el Perú? Gac Sanit. 2016;30(2).Disponible en http://scielo.isciii.es/scielo.php?script=sci_ arttext\&pid=S0213-91112016000200016 [Revisado 26 febrero 2020].

5. Novoa A. De cómo corrompe la industria a los médicos. [Online]. Disponible en http://www. nogracias.eu/2013/03/20/de-como-corrompe-la-industria-a-los-medicos/ [Revisado 26 febrero 2020].

6. Boletín Oficial del País Vasco. Orden de la consejera de Salud, por la que se regula la visita médica de los delegados de la industria farmacéutica y de representantes de fabricantes, distribuidores o comercializadores de productos y tecnologías sanitarias, en las organizaciones de servicios sanitarios del Sistema Sanitario de Euskadi. Disponible en https://www.euskadi. eus/gobierno-vasco/-/eli/es-pv/o/2020/02/10/ (1)/dof/spa/html/ [Revisado 26 febrero 2020]

7. Salas $\mathrm{S}$ et al. Conflictos de intereses en la práctica clínica. Análisis ético de algunas relaciones con la industria. Rev Méd Chile 2006;134:1576-82. Disponible en https:// scielo.conicyt.cl/scielo.php?script=sci_arttext\&pid=S0034-98872006001200014 [Revisado 26 febrero 2020].

8. Comisión Central de Deontología de la Organización Médica Colegial (OMC). España 2000-2015. Disponible en https://www.acta- sanitaria.com/wp-content/uploads/2015/11/ declaraciones-de-la-comision-central-de-deontologia-2000-2015.pdf. [Revisado 26 de febrero 2020].

9. Altisent $\mathrm{R}$, et al. Conflictos de interés en la profesión médica. Aten Primaria. 2019. Disponible en https://doi.org/10.1016/j.aprim.2019.05.004 [Revisado 26 febrero 2020].

10. Gómez C AI, Latorre S C, Carreño J. Dilemas éticos en las relaciones entre la industria farmacéutica y los profesionales de la salud. P \& B. Revista N¹. 2007;11(28):23-38 Disponible en http://www.scielo.org.co/pdf/pebi/v11n1/ v11n1a03.pdf [revisado 26 febrero 2020]

11. Martin Moreno S. Ética de la prescripción. Conflictos del médico con el paciente, la entidad gestora y la industria farmacéutica. Medicina Clínica. 2001;116(8). Disponible en http://aulavirtual.farmacoterapia.sanidadmadrid.org/UPC_VirtualCampus/Contents/ Course/Actualizacion_pediatria2/curso/02/ pdf/etica.pdf. [Revisado 26 febrero 2020]

12. González-Rubio R et al. Industria farmacéutica y formación sanitaria especializada: percepciones de los MIR en Madrid Gac Sanit. 2019;33(2):112-8. Disponible en https:// www.sciencedirect.com/science/article/pii/ S0213911117301954. [Revisado 28 febrero 2020]

13. Galán Herrera $S$, et al. Análisis de la relación entre el médico de atención primaria y la industria farmacéutica. Aten Primaria 2004;34(5):231-47. Disponible en https:// www.elsevier.es/es-revista-atencion-primaria-27-articulo-analisis-relacion-entre-el-medico-13066401. [Revisado 28 febrero 2020]. 
14. Buffo-Sequeira I, Arroyo-Castelán E, Halabe-Cherem J, Monroy-Saint Martin M. El médico y la relación con la industria farmacéutica Revista CONAMED. 2012;17(4). Disponible en file://C:/Users/Rafael\%20J\%202/ Downloads/Dialnet-ElMedicoYLaRelacionConLaIndustriaFarmaceutica-4175687.pdf
15. Florián-Castro RO. Percepción sobre promoción farmacéutica y prescripción de medicamentos en médicos de Lima, Perú 2016. Rev. Perú. med. exp. salud pública. 2018;35(4). Disponible en http://www.scielo.org.pe/scielo.php?script=sci_ arttext\&pid=S1726-46342018000400026\&ln$\mathrm{g}=\mathrm{es} \& \mathrm{nrm}=$ iso [Revisado 28 de febrero 2020]. 